\title{
Dimensional Reduction in Evolving Spin-Glass Model: Correlation of Phenotypic Responses to Environmental and Mutational Changes
}

\author{
Ayaka Sakata๑* \\ Department of Statistical Inference \& Mathematics, Institute of Statistical Mathematics, \\ 10-3 Midori-cho, Tachikawa, Tokyo 190-8562, Japan \\ Kunihiko Kaneko뭉 \\ Center for Complex Systems Biology, Universal Biology Institute, University of Tokyo, \\ 3-8-1 Komaba, Meguro-ku, Tokyo 153-8902, Japan
}

(Received 11 January 2020; accepted 5 May 2020; published 26 May 2020)

\begin{abstract}
The evolution of high-dimensional phenotypes is investigated using a statistical physics model consisting of interacting spins, in which phenotypes, genotypes, and environments are represented by spin configurations, interaction matrices, and external fields, respectively. We found that phenotypic changes upon diverse environmental change and genetic variation are highly correlated across all spins, consistent with recent experimental observations of biological systems. The dimension reduction in phenotypic changes is shown to be a result of the evolution of the robustness to thermal noise, achieved at the replica symmetric phase.
\end{abstract}

DOI: 10.1103/PhysRevLett.124.218101

Biological systems generally consist of a huge number of components. Biomolecules (proteins) consist of a large number of monomers (amino acids), whereas cells consist of a variety of proteins, mRNAs, and other chemicals. Despite such high dimensionality, however, there is growing evidence that the responses of phenotypes to external changes are often restricted to a low-dimensional subspace.

For instance, the concentrations of a huge variety of components such as mRNAs and proteins have been recently measured against a variety of environmental stresses. The changes in the (logarithmic) concentrations of mRNAs or proteins are found to be correlated [1-3] or proportional [4-6] across all components, against a variety of environmental stresses. This global proportionality suggests that phenotypic changes against environmental perturbations are constrained along a one- or low-dimensional manifold, a manifestation of a drastic dimension reduction from the high-dimensional composition space $[7,8]$. Indeed, such dimension reduction would be rather universal in biological systems, as reported in studies of protein dynamics [9], ecological systems [10], and neural learning dynamics [11]. This global proportional change is also extended to the evolutionary dimension. Changes in each concentration upon genetic mutation and those upon

Published by the American Physical Society under the terms of the Creative Commons Attribution 4.0 International license. Further distribution of this work must maintain attribution to the author(s) and the published article's title, journal citation, and DOI. environmental perturbations are also highly correlated [12-16]. It has been recently conjectured that such dimension reduction is a consequence of the evolution to achieve functional phenotypes that are robust to perturbations. Although some evolution simulations of catalytic-reaction networks support this conjecture [7,17], thus far the concept remains an intuitive sketch, and an underlying mathematical structure remains elusive.

At this moment, a statistical physics approach would be useful to address the question of if and how the dimension reduction evolves. Previously, we studied a statistical physics model of spins, whose stochastic change is governed by a Hamiltonian that includes the two-body spin-spin interaction $J_{i j}$ under thermal noise, specified by the temperature $[18,19]$. In the model, the following correspondences are taken: phenotypes $\rightarrow$ spin configurations $\left\{S_{i}\right\}$; rule to shape the phenotype $\rightarrow$ Hamiltonian for spin-spin interaction $H=-\sum_{i, j} J_{i j} S_{i} S_{j}$; environmental condition $\rightarrow$ external field $h_{i}$ to each spin in the Hamiltonian. The evolution process is introduced by the "mutation" in $J_{i j}$ and a selection according to the fitness defined from the spin configuration. By evolving the Hamiltonian under a certain temperature, we have previously demonstrated the evolution of Hamiltonians to shape phenotypes to be robust to perturbations at an intermediate temperature corresponding to the replica-symmetric (RS) phase, whereas replica symmetry breaking (RSB) at lower temperature leads to a rugged energy landscape and a nonrobust phenotype. Still, the dimension reduction and its relationship with these phases was not investigated, which is one of the main focuses of the present Letter. 
By taking advantage of this spin model and evolving it under a certain temperature, one can investigate if the dimension reduction in phenotypic changes, as observed in biological systems, is formulated and understood in terms of statistical physics. Specifically, we focus on the following questions: (i) Are high-dimensional phenotypic changes against various environmental changes correlated? (ii) Are the changes induced by environmental and genetic changes correlated? (iii) If the above two correlations are observed, are they a result of dimension reduction from a highdimensional phenotypic space, shaped by evolution? (iv) Finally, within what range of temperature are the above questions answered affirmatively? In other words, is the appropriate noise relevant to the evolution of dimension reduction? By answering these questions, we will elucidate the origin of dimension reduction in terms of statistical physics, in possible relationship with RS/RSB.

Now, we define a spin-statistical physics model for phenotypic evolution, in which the phenotype is denoted by spins $\boldsymbol{S}=\left[S_{1}, \ldots, S_{N}\right] \in\{-1,+1\}^{N}$. The dynamics of the spins are given by the stochastic dynamics, prescribed by the Hamiltonian $H$ as

$$
H(\boldsymbol{S} \mid \boldsymbol{J})=-\frac{1}{2} \boldsymbol{S}^{\mathrm{T}} \boldsymbol{J} \boldsymbol{S}
$$

where superscript $\mathrm{T}$ denotes the transpose, and $\boldsymbol{J} \in \mathbb{R}^{N \times N}$ is a symmetric matrix whose diagonal components are zero. With this Hamiltonian, the spin dynamics with discrete time $t$ is given by the transition probability

$$
\operatorname{Pr}\left[\boldsymbol{S}^{(t)} \rightarrow \boldsymbol{S}^{(t+1)} \mid \boldsymbol{J}\right]=\min \left\{e^{-\beta \Delta H\left(\boldsymbol{S}^{(t)}, \boldsymbol{S}^{(t+1)} \mid \boldsymbol{J}\right)}, 1\right\},
$$

where $\boldsymbol{S}^{(t)}$ is the phenotype at step $t$, and $\Delta H\left(\boldsymbol{S}, \boldsymbol{S}^{\prime} \mid \boldsymbol{J}\right) \equiv$ $H\left(\boldsymbol{S}^{\prime} \mid \boldsymbol{J}\right)-H(\boldsymbol{S} \mid \boldsymbol{J})$. Here, $\boldsymbol{S}^{(t+1)}$ differs from $\boldsymbol{S}^{(t)}$ only by a single site, hence spin configuration is asynchronously updated. The inverse temperature $\beta=T^{-1}$ describes the stochasticity of the phenotype expression process. The elements of the interaction matrix are chosen as $J_{i j} \in$ $\Omega_{J}(i \neq j)$ with $\Omega_{J}=\{-1 / \sqrt{N}, 0,1 / \sqrt{N}\}$, and $J_{i i}=0$ $(i=1, \ldots, N)$. This matrix represents the genotype, which evolves over generations, as will be described later.

The fitness is generally given as a function of phenotypes, i.e., the spin configuration. Here, we assume that a part of the spins, named targets $i \in \mathcal{T}$, contributes to the fitness, such as the active site residues of protein. As more of the target spins have the same value +1 or -1 , the fitness $\psi(\boldsymbol{J})$ is higher, as defined as

$$
\psi(\boldsymbol{J})=\overline{\left|m_{\mathcal{T}}\right|}, \quad m_{\mathcal{T}}=\frac{1}{N_{T}} \sum_{i \in \mathcal{T}} S_{i},
$$

where $N_{T}$ is the size of $\mathcal{T}$, and $\cdots$ denotes the average over the trajectories of the phenotype expression dynamics, which depend on genotype $\boldsymbol{J}$.
The evolution to select genotypes with higher fitness is represented by the following stochastic update rule with discrete time,

$$
\operatorname{Pr}\left[\boldsymbol{J}^{(g)} \rightarrow \boldsymbol{J}^{(g+1)}\right]=\min \left\{e^{\beta_{J} \Delta \psi\left(\boldsymbol{J}^{(g)} \boldsymbol{J}^{(g+1)}\right)}, 1\right\},
$$

where $\Delta \psi\left(\boldsymbol{J}^{\prime}, \boldsymbol{J}\right)=\psi\left(\boldsymbol{J}^{\prime}\right)-\psi(\boldsymbol{J})$. The parameter $\beta_{J}=T_{J}^{-1}$ represents the selection pressure; as $T_{J}$ decreases, the genotype with higher fitness survives to the next generation with high probability.

We mainly describe the results for $N=100$ and $\rho \equiv$ $N_{T} / N=0.05$, unless otherwise mentioned. For the phenotype dynamics Eq. (2), we adopt the Markov Chain Monte Carlo (MCMC) method with detailed balance condition. After a sufficient number of updates, the distribution of $\boldsymbol{S}$ is expected to converge to the equilibrium distribution, $P(\boldsymbol{S}) \propto \exp [-\beta H(\boldsymbol{S} \mid \boldsymbol{J})]$, for a given genotype. We numerically calculated the thermal average over $t_{f}=2 \times 10^{4} \mathrm{MC}$ steps, after discarding the initial $t_{\mathrm{i}}=10^{4}$ steps.

At each generation $g$, the candidates of genotype $\boldsymbol{J}^{(g+1)}$ are generated by introducing the mutations with probability $p_{\mu}=0.05$; hence $\boldsymbol{J}^{(g+1)}$ differs from $\boldsymbol{J}^{(g)}$ by $0.05 \times N(N-1) / 2$ components [20]. The values of $J_{i j}$ $(i \neq j)$ change into one of the components in $\Omega_{J} \backslash J_{i j}$ with equal probability, where $A \backslash a$ denotes the members of $A$, excluding $a$. We numerically update genotypes over generation $g_{\max }=10^{5}$ at $T_{J}=0.05$ [21]. Without a loss of generality, hereafter, we set the target sites as $\mathcal{T}=$ $\left\{1, \ldots, N_{T}\right\}$. We numerically obtain 100 genotypes evolved at $\rho$ and $T$ with different initial conditions, and the set is denoted as $\mathcal{J}(T)$.

First, we present the existence of three phases that depend on $T$ [18,19]. Figure 1(a) shows the temperature dependence of the averaged fitness over $\mathcal{J}(T)$. At $T \geq T_{c 2}$, the fitness value approaches 0.375 as $T$ increases, which is the level expected by the random spin configuration [22]. Hence, the phase $T \geq T_{c 2}$ is identified as the paramagnetic phase. The high-fitness phase is separated into two phases at $T=T_{c 1}$. The region at $T_{c 1} \leq T<T_{c 2}$ is the RS phase, as is characterized by the convergence of the belief propagation (BP) algorithm [23] [24]. The fitted state is reached fast enough and is robust to noise and mutation. As shown in Fig. 1(b), the fraction of $\boldsymbol{J} \in \mathcal{J}(T)$, in which the BP algorithm does not converge within $10^{5}$ steps, increases from zero at $T_{c 1}$. Hence, the phase at $T<T_{c 1}$ corresponds to the RSB phases, as characterized by the rugged energy landscape (see also Ref. [18]).

Now, we discuss if the response to different environmental conditions is correlated or not, depending on the phase. Hereafter, we study the symmetry breaking local magnetization $\mu_{i}=\overline{\operatorname{sign}\left(m_{\mathcal{T}}\right) S_{i}}$, considering the $\mathrm{Z}_{2}$ symmetry [27]. Under the infinitesimal external fields, the difference between expression patterns $\delta \mu_{i}^{(h)}(\boldsymbol{h}, \boldsymbol{J} ; \delta \boldsymbol{h}) \equiv$ $\mu_{i}(\boldsymbol{h}+\delta \boldsymbol{h}, \boldsymbol{J})-\mu_{i}(\boldsymbol{h}, \boldsymbol{J})$ is expanded as 

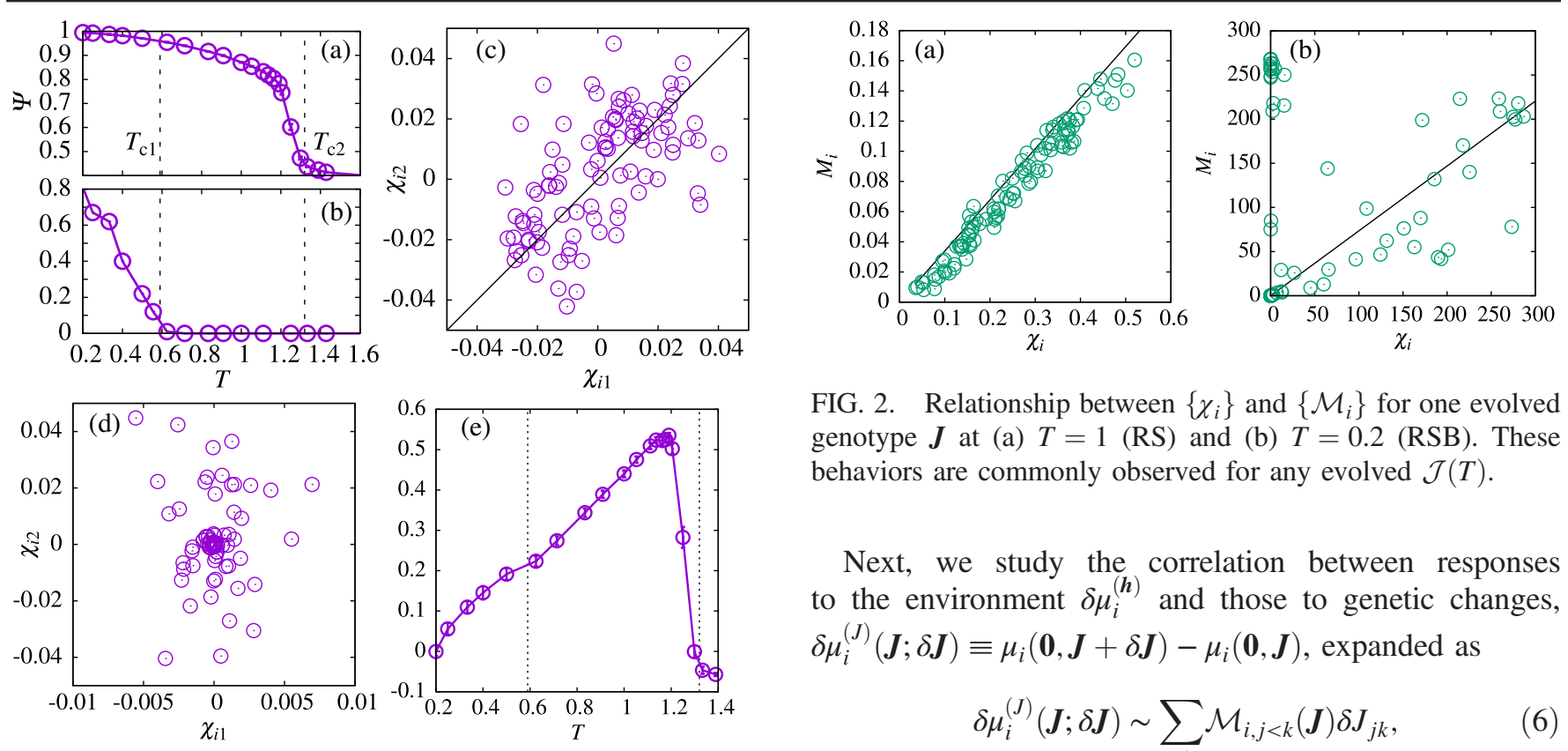

FIG. 1. (a) $T$ dependence of the averaged fitness. (b) Fraction of matrices $\mathcal{J}(T)$ in which the BP algorithm does not converge within $10^{5}$ steps. For (a) and (b), the vertical dashed lines indicate the phase transition temperatures. (c)-(d) Scatter plots of $\chi_{i 1}$ and $\chi_{i 2}$ at (c) $T=1$ and (d) $T=0.4$. The slope of the diagonal line in (c) is 1 . (e) $T$ dependence of the averaged correlation coefficients between $\left\{\chi_{i 1}\right\} \cdots\left\{\chi_{i, N_{T}}\right\}$. The statistical errors over $\mathcal{J}(T)$ is smaller than the point size in the figure for all $T$ region, and error bars are not discernible.

$$
\delta \mu_{i}^{(h)}(\boldsymbol{h}, \boldsymbol{J} ; \delta \boldsymbol{h}) \sim \sum_{j} \chi_{i j}(\boldsymbol{h}, \boldsymbol{J}) \delta h_{j},
$$

where $\chi_{i j}(\boldsymbol{h}, \boldsymbol{J})=\partial \mu_{i}(\boldsymbol{h}, \boldsymbol{J}) / \partial h_{j}$ is the susceptibility. We regard Eq. (5) as the response of the $i$ th component to the additional external field, for a system with genotype $\boldsymbol{J}$ subject to external field $\boldsymbol{h}$. For simplicity, we consider the case that an external field $\delta \boldsymbol{h}_{i}$, whose $i$ th component is $\delta h(\neq 0)$, otherwise 0 , is applied to the system at $\boldsymbol{h}=\mathbf{0}$. The first-order response of the $j$ th component to $\delta \boldsymbol{h}_{i}$ is $\chi_{j i}(\mathbf{0}, \boldsymbol{J})$. At the equilibrium, $\chi_{i j}(\boldsymbol{h}, \boldsymbol{J})=\beta\left(\left\langle S_{i} S_{j}\right\rangle_{\boldsymbol{h}}-\left\langle S_{i}\right\rangle_{\boldsymbol{h}}\left\langle S_{j}\right\rangle_{\boldsymbol{h}}\right)$ holds, where $\langle\cdot\rangle_{h}$ means the average according to the equilibrium distribution under the external field $\boldsymbol{h}$; $P(\boldsymbol{S}) \propto \exp \left(-\beta H(\boldsymbol{S} \mid \boldsymbol{J})+\beta \boldsymbol{h}^{\mathrm{T}} \boldsymbol{S}\right)$. We numerically compute $\chi_{i j}$ by MCMC simulation as $\chi_{i j}=\beta\left(\overline{S_{i} S_{j}}-\mu_{i} \mu_{j}\right)$. Figure 1 shows the scatter plots of $\chi_{i 1}$ and $\chi_{i 2}$ under one realized genotype for $i \geq 3$ at (c) $T=1$ (RS) and (d) $T=0.4$ (RSB). Their correlation coefficients are (c) 0.59 , and (d) 0.035 , respectively. Here, we ignore the responses of $\mu_{1}$ and $\mu_{2}$ to remove the trivial strong response directly to $\delta \boldsymbol{h}_{1}$ and $\delta \boldsymbol{h}_{2}$ itself. In Fig. 1(e), $T$ dependence of the correlation coefficient between $\left\{\chi_{i 1}\right\} \cdots\left\{\chi_{i, N_{T}}\right\}$ is shown, which is averaged over $\mathcal{J}(T)$. The correlation between the responses to external fields $\delta \boldsymbol{h}_{i}(i \in \mathcal{T})$ is discernible in the RS phase [28].

FIG. 2. Relationship between $\left\{\chi_{i}\right\}$ and $\left\{\mathcal{M}_{i}\right\}$ for one evolved genotype $\boldsymbol{J}$ at (a) $T=1$ (RS) and (b) $T=0.2$ (RSB). These behaviors are commonly observed for any evolved $\mathcal{J}(T)$.

Next, we study the correlation between responses to the environment $\delta \mu_{i}^{(\boldsymbol{h})}$ and those to genetic changes, $\delta \mu_{i}^{(J)}(\boldsymbol{J} ; \delta \boldsymbol{J}) \equiv \mu_{i}(\mathbf{0}, \boldsymbol{J}+\delta \boldsymbol{J})-\mu_{i}(\mathbf{0}, \boldsymbol{J})$, expanded as

$$
\delta \mu_{i}^{(J)}(\boldsymbol{J} ; \delta \boldsymbol{J}) \sim \sum_{j k} \mathcal{M}_{i, j<k}(\boldsymbol{J}) \delta J_{j k},
$$

where $\mathcal{M}_{i, j k}=\partial \mu_{i}(\boldsymbol{J}) / J_{j k}$, which corresponds to $\beta\left(\left\langle S_{i} S_{j} S_{k}\right\rangle-\left\langle S_{i}\right\rangle\left\langle S_{j} S_{k}\right\rangle\right)$ at the equilibrium. For the comparison between $\delta \mu_{i}^{(h)}$ and $\delta \mu_{i}^{(J)}(\boldsymbol{J})$, we assume that the components of $\delta \boldsymbol{h}$ and $\delta \boldsymbol{J}$ independently follow a Gaussian distribution with mean 0 and variance $\epsilon$ for $\delta \boldsymbol{h}$, and variance $\epsilon / \sqrt{N}$ for $\delta \boldsymbol{J}$, respectively. The expected squared responses are given by

$$
\begin{aligned}
E_{\delta \boldsymbol{h}}\left[\delta \mu_{i}^{(h) 2}(\boldsymbol{h}, \boldsymbol{J} ; \delta \boldsymbol{h})\right] & \simeq \epsilon^{2} \chi_{i}(\boldsymbol{h}, \boldsymbol{J}), \\
E_{\delta \boldsymbol{J}}\left[\delta \mu_{i}^{(J) 2}(\boldsymbol{J} ; \delta \boldsymbol{J})\right] & \simeq \epsilon^{2} \mathcal{M}_{i}(\boldsymbol{J}),
\end{aligned}
$$

where $E_{\delta \boldsymbol{h}}[\cdot]$ and $E_{\delta J}[\cdot]$ denote the average over $\delta \boldsymbol{h}$ and $\delta \boldsymbol{J}$, respectively, and $\chi_{i}=\sum_{j \neq i} \chi_{i j}^{2}$, and $\mathcal{M}_{i}=$ $N^{-1} \sum_{j<k, j, k \neq i} \mathcal{M}_{i, j k}^{2}$. The quantities $\chi_{i}(\boldsymbol{h}, \boldsymbol{J})$ and $\mathcal{M}_{i}(\boldsymbol{J})$ correspond to the spin-glass susceptibility and "susceptibility to interaction matrix," and indicate the sensitivity of the $i$ th component to the external field and mutation, respectively. Figure 2 shows the scatter plot between $\mathcal{M}_{i}(\boldsymbol{J})$ and $\chi_{i}(\mathbf{0}, \boldsymbol{J})$ for genotype $\boldsymbol{J} \in \mathcal{J}(T)$ at (a) $T=1$ (RS) and (b) $T=0.2$ (RSB). A linear relationship between $\chi_{i}$ and $\mathcal{M}_{i}$ arises in the RS phase.

These numerical simulations indicate that the evolution under thermal fluctuation that leads to the RS phase induces the correlations between the responses. To understand the emergence of the correlation, we decompose the evolved genotypes into eigenvalues and eigenvectors as $\boldsymbol{J}=\boldsymbol{\Xi} \boldsymbol{\Lambda} \boldsymbol{\Xi}^{\mathrm{T}}$, where $\boldsymbol{\Lambda} \in \mathbb{R}^{N \times N}$ is a diagonal matrix consisting of eigenvalues $\Lambda_{i i}=\lambda_{i} \quad\left(\lambda_{1} \geq \lambda_{2} \geq \cdots \geq \lambda_{N}\right)$, and $\boldsymbol{\Xi}=$ $\left[\boldsymbol{\xi}^{1}, \ldots, \boldsymbol{\xi}^{N}\right] \in \mathbb{R}^{N \times N}$ is the set of corresponding eigenvectors. Figure 3(a) shows the averaged values of the first and second eigenvalues over $\boldsymbol{J} \in \mathcal{J}(T)$. The first eigenvalue is much larger in the RS phase than those in the other phases. 

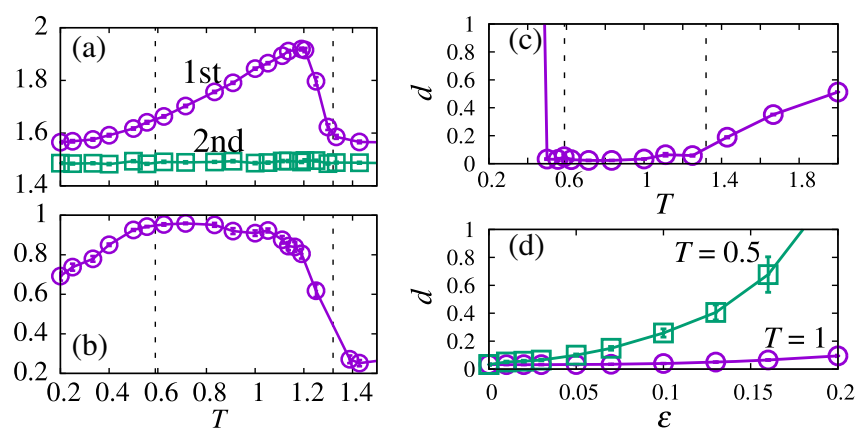

FIG. 3. $\quad T$ dependence of (a) averaged first and second eigenvalues of $\boldsymbol{J}$, (b) correlation coefficient between $\operatorname{arctanh}\left(\mu_{i}\right)$ and $\xi_{i}^{1}$, and (c) averaged $d$. Vertical dashed line denotes transition temperatures. (d) $\epsilon$ dependence of $d$ for $T=1$ (RS) and $T=0.5(\mathrm{RSB})$.

The evolutionary change of the second eigenvalue is vanishingly small for any $T$. This tendency is common for any $\lambda_{i}(i \geq 2)$. Hence, the dominancy of the first eigenmode is enforced as a result of the evolution at $T_{c 1} \leq T<T_{c 2}$.

On the basis of the large contribution of the first eigenvalue in the RS phase, we apply a 1-rank approximation of genotype $\boldsymbol{J} \sim \eta_{1} \xi^{1} \xi^{1 \mathrm{~T}}$. By a straightforward calculation, the local magnetization is expressed as

$$
\mu_{i}=\tanh \left(\beta \eta_{1} \xi_{i}^{1} \sum_{k \neq i} \xi_{k}^{1} \mu_{k}+h_{i}\right),
$$

at sufficiently large $N$. Therefore, when the first eigenmode is dominant, the relationship $\xi_{i}^{1} \propto \operatorname{atanh}\left(\mu_{i}\right)$ should hold at $\boldsymbol{h}=\mathbf{0}$. Figure 3 (b) shows the correlation coefficient between $\left\{\operatorname{atanh}\left(\mu_{i}\right)\right\}$ and $\left\{\xi_{i}^{1}\right\}$. In the RS phase, the correlation coefficient approaches 1 ; hence, $\xi_{i}^{1} \sim \operatorname{atanh}\left(\mu_{i}\right)$ is a reasonable approximation. We note that the expression of $\boldsymbol{J}=$ $\eta_{1} \xi^{1} \xi^{1 \mathrm{~T}}$ is similar to those of the Mattis model [29,30], which is the Hopfield model with a single embedded pattern [31]. The present embedded pattern, however, is $\sqrt{\eta_{1}} \xi^{1}$, in contrast to a discrete vector with \pm 1 in the Mattis model. For sufficiently small $\rho$, the distribution of $\mu_{i}$ is almost random, and the embedded pattern after the evolution is a random pattern, except the target spins [32]. Even though the approximate estimate by Mattis-type model is used here, the evolved genotypes in the RS phase do not perfectly agree with it: Indeed, eigenmodes other than the first mode remain, which induces frustration between nontarget spins [18]. This hampers the correlation between responses of nontarget spins.

Last, we show that the dominancy of the first eigenmode of genotype induces a correlation between the responses to environmental and genetic changes, as observed in the RS phase. From Eq. (9), we obtain the expression for susceptibility under the 1-rank approximation

$$
\chi_{i j}=v_{i}\left(\delta_{i j}+\eta_{1} \xi_{i}^{1} \sum_{k \neq i} \xi_{k}^{1} \chi_{k j}\right),
$$

where $v_{i}=\beta\left(1-\mu_{i}^{2}\right)$ and $\delta_{i j}$ is Kronecker's delta. Because of the randomness of the embedded pattern, it is reasonable to assume that $\chi_{i j}(i \neq j)$ is sufficiently small; hence, $\left\langle S_{i} S_{j}\right\rangle \sim \mu_{i} \mu_{j}$ holds. Applying the equilibrium relationship $\mathcal{M}_{i, j k}=\partial\left\langle S_{j} S_{k}\right\rangle / \partial h_{i}$, we obtain $\mathcal{M}_{i, j k} \sim \chi_{i j} \mu_{k}+\mu_{j} \chi_{i k}$. Because $\left\{\mu_{i}\right\}$ is expected to be randomly distributed, $\mathcal{M}_{i}=\sum_{j k} \chi_{i j}^{2} \mu_{k}^{2}$ holds, neglecting the cross term. Setting $Q \equiv N^{-1} \sum_{i} \mu_{i}^{2}$, we obtain

$$
\mathcal{M}_{i}(\boldsymbol{J})=\chi_{i}(\mathbf{0}, \boldsymbol{J}) Q .
$$

Hence, the proportionality between $\left\{\chi_{i}\right\}$ and $\left\{\mathcal{M}_{i}\right\}$ is a consequence of the dominance of the first eigenmode evolved in the RS phase, i.e., the evolutionary dimensional reduction. Here, notice that for the Mattis system, Eq. (11) itself holds but $\mathcal{M}_{i}$ and $\chi_{i}$ are not distributed and take unique values over all $i$, hence the proportionality between the distributed $\mathcal{M}_{i}$ and $\chi_{i}$ as in Fig. 2 is not observed. The distribution comes from the nontarget spins in our model.

The relationship Eq. (11) is indicated by the solid line in Fig. 2. We quantify the deviation of the observed $\chi$ - $\mathcal{M}$ relationship from the theoretical line Eq. (11), by the normalized mean squared error $d=\sum_{i}\left(\mathcal{M}_{i}-Q \chi_{i}\right)^{2} / \sum_{i} \mathcal{M}_{i}^{2}$. Figure 3(c) shows the $T$ dependence of $d$ averaged over $\mathcal{J}(T)$. In the RS phase, $d$ is close to 0; hence, Eq. (11) holds with high accuracy, which is a result of the emergence of the dominant first eigenmodes, accompanied by randomness in the nontarget spins.

When $T\left(<T_{c 1}\right)$ is close to the RS-RSB boundary, $d$ is close to 0 , as with the RS phase. The difference between the $\mathrm{RS}$ and RSB phase is clear for finite $\boldsymbol{h}$ and $\Delta \boldsymbol{J}$, which is a deviation of $\boldsymbol{J}$ from $\mathcal{J}(T)$. We randomly generate $\boldsymbol{h} \sim$ $\mathcal{N}\left(\mathbf{0}, \epsilon^{2} \boldsymbol{I}\right)$ and symmetric $\Delta \boldsymbol{J}$, where $\Delta J_{i j} \sim \mathcal{N}\left(0, \epsilon^{2} / N\right)$, and $\Delta J_{i i}=0 \forall i$. We quantify the relationship between $\chi_{i}(\boldsymbol{h}, \boldsymbol{J})$ and $\mathcal{M}_{i}(\boldsymbol{J}+\Delta \boldsymbol{J})$ using $d$. Figure 3(d) shows $\epsilon$ dependence of the averaged $d$ over $\mathcal{J}(T)$ and 100 samples of $\boldsymbol{h}$ and $\Delta \boldsymbol{J}$ for $T=1$ (RS) and $T=0.5$ (RSB). In the RSB phase, $d$ increases faster than it does in the RS phase, even when $d$ at $\epsilon=0$ is close to zero. This robustness of the proportionality is also a consequence of the dominant first eigenmode [32].

The proportionality between $\left\{\chi_{i j}\right\}$ and $\left\{\chi_{i k}\right\}\left(j, k \leq N_{T}\right.$, $j \neq k$ ), shown in Fig. 1(b), is also a consequence of the dominant first eigenmode. From Eq. (10), the leading term of susceptibility is $\chi_{i j}=\eta_{1} \xi_{i}^{1} \xi_{j}^{1} v_{i} v_{j}(i \neq j)$; hence, $\chi_{i j} / \chi_{i k}=\xi_{j}^{1} v_{j} /\left(\xi_{k}^{1} v_{k}\right)$. In the RS phase, both $v_{j}$ and $\xi_{j}^{1}$ are functions of $\mu_{j}$; hence, $\chi_{i j} / \chi_{i k} \sim 1$ holds when $\mu_{j} \simeq \mu_{k}$. This is the origin of the linear relationship between $\left\{\chi_{i j}\right\}$ and $\left\{\chi_{i k}\right\}$ [33].

In summary, we applied an evolving spin-statistical physics model, representing phenotypes, genotypes, 
and the environment by spin configuration, interaction matrix, and the external field, respectively, and have answered the questions addressed at the beginning of this Letter. (i) Correlated responses across different environmental changes are demonstrated by the correlation in susceptibilities $\chi_{i j}$ and $\chi_{i \ell}$ in the evolved genotypes at the RS phase. (ii) Proportional responses to mutation and environmental changes are demonstrated by the proportionality between the "susceptibility to interaction matrix" $\mathcal{M}_{i}$ and spin-glass susceptibility $\chi_{i}$. (iii) These proportional responses originate in the reduction of rank in the interaction matrix. (iv) Such dimension reduction and proportional changes are observed for the evolved genotypes at the RS phase, i.e., at an intermediate level of thermal noise. The RS phase was also evolved in a fully connected system, where the frustration around target spins is diminished, and termed as the local Mattis state [18]. The current study demonstrates that such a RS phase (in a sparse connection) shows the correlated responses of phenotypes to environment and mutation, with dimension reduction, as supported by the redundant degrees of freedom by nontarget spins.

Hence, robustness of phenotypes to noise [34,35] is essential to the evolutionary dimension reduction, leading to the correlated responses in the high-dimensional phenotypes to different types of perturbations. Although the present statistical physics model is highly simplified, it gives a theoretical basis for dimension reduction in biological systems, in which robustness to noise is also essential. In fact, the present model can be interpreted as the evolution of protein to have a certain function. The RS phase here corresponds to the funnel structure in contrast to the spin-glass phase [36]. Note that recent reports on protein dynamics suggest the existence of large collective motion, which may be a manifestation of dimension reduction [9,37-39]. The correspondence between noise and mutation responses is also consistent with the simulation [12] and experiments [40] of the evolution of t-RNA. Last, although dynamics at the cellular level are not represented by a Hamiltonian, the similarity between spin-glass dynamics and gene expression dynamics with mutual activation and inhibition is now well recognized $[34,35,41-43]$. In these examples, correlated phenotypic responses as a result of dimension reduction are evolutionarily acquired as in the RS phase in our model at an intermediate temperature.

In terms of statistical physics, the evolution to the RS phase under appropriate levels of noise should be considered, in which both higher fitness and robustness to noise are achieved with the dimension reduction. If the temperature is reduced, robustness in the phenotype is lost by $\mathrm{RSB}$, even though a higher fitness state is reached after sufficient time steps of expression. Here, we have studied the simplest fitness condition. For higher biological functions, the response to diverse environmental conditions, say, different target spin configurations upon the application of different external fields, may be required. The extension to such problems would be straightforward, in which the need for both robustness and plasticity may lead to dimension reduction with higher ranks.

The authors thank Koji Hukushima and Yoshiyuki Kabashima for helpful comments and discussions. This research was partially supported by a Grant-in-Aid for Scientific Research (S) (15H05746) and (A) (20H00123) from the Japanese Society for the Promotion of Science (JSPS) and Grant-in-Aid for Scientific Research on Innovative Areas (17H06386) from the Ministry of Education, Culture, Sports, Science and Technology (MEXT) of Japan.

*ayaka@ism.ac.jp

[1] S. Bergmann, J. Ihmels, and N. Barkai, PLoS Biol. 2, E9 (2003)

[2] T. S. Gunasekera, L. N. Csonka, and O. Paliy, J. Bacteriol. 190, 3712 (2008).

[3] S. Marguerat, A. Schmidt, S. Codlin, W. Chen, R. Aebersold, and J. Bähler, Cell 151, 671 (2012).

[4] Y. Matsumoto, Y. Murakami, S. Tsuru, B. Y. Ying, and T. Yomo, BMC Genomics 14, 808 (2013).

[5] K. Kaneko, C. Furusawa, and T. Yomo, Phys. Rev. X 5, 011014 (2015)

[6] A. Schmidt, K. Kochanowski, S. Vedelaar, E. Ahrné, B. Volkmer, L. Callipo, K. Knoops, M. Bauer, R. Aebersold, and M. Heinemann, Nat. Biotechnol. 34, 104 (2016).

[7] C. Furusawa and K. Kaneko, Phys. Rev. E 97, 042410 (2018).

[8] K. Kaneko and C. Furusawa, Annu. Rev. Biophys. 47, 273 (2018).

[9] T. Tlusty, A. Libchaber, and J. P. Eckmann, Phys. Rev. X 7, 021037 (2017).

[10] Z. Frentz, S. Kuehn, and S. Leibler, Phys. Rev. X 5, 041014 (2015).

[11] P. T. Sadtler, K. M. Quick, M. D. Golub, S. M. Chase, S. I. Ryu, E. C. Tyler-Kabara, M. Y. Byron, and A.P. Batista, Nature (London) 512, 423 (2014).

[12] L. W. Ancel and W. Fontana, J. Exp. Zool. B 288, 242 (2000).

[13] V. Pancaldi, F. Schubert, and J. Bahler, Mol. Biosyst. 6, 543 (2010).

[14] C. Furusawa and K. Kaneko, J. R. Soc. Interface 12, 20150482 (2015).

[15] T. Horinouchi, K. Tamaoka, C. Furusawa, N. Ono, S. Suzuki, T. Hirasawa, T. Yomo, and H. Shimizu, BMC Genomics 11, 579 (2010).

[16] T. Horinouchi, S. Suzuki, T. Hirasawa, N. Ono, T. Yomo, H. Shimizu, and C. Furusawa, BMC Evol. Biol. 15, 180 (2015).

[17] T. U. Sato and K. Kaneko, Phys. Rev. Research 2, 013197 (2020).

[18] A. Sakata, K. Hukushima, and K. Kaneko, Phys. Rev. Lett. 102, 148101 (2009). 
[19] A. Sakata, K. Hukushima, and K. Kaneko, Europhys. Lett. 99, 68004 (2012).

[20] We have confirmed that $p_{\mu}=0.05$ does not change the ensemble $\mathcal{J}(T)$ that is obtained by the asynchronous update of $\boldsymbol{J}$.

[21] This choice of $T_{J}$ is appropriate to investigate the $T$ dependence of evolved genotypes. For the $T_{J}$ dependence, see also Ref. [19].

[22] The target-spin configurations here have 3 possibilities: (i) 5 spins aligned, $\left|m_{\mathcal{T}}\right|=1$, with probability $2 / 32$; (ii) 4 spins aligned, $\left|m_{\mathcal{T}}\right|=0.6$, with probability $10 / 32$; (iii) 3 spins aligned, $\left|m_{\mathcal{T}}\right|=0.2$, with probability $20 / 32$. The summation of these leads to $\psi=3 / 8$.

[23] M. Mézard and A. Montanari, Information, Physics, and Computation (Oxford University Press, Oxford, 2009).

[24] In the fully connected system, the stability condition of the $\mathrm{BP}$ algorithm agrees with the validity of the RS assumption, which is known as de Almeida-Thouless (AT) instability $[25,26]$. The BP algorithm is generally adopted as a numerical method to judge the RSB transition for a not fully connected system, where analytical derivation of the AT instability is not available.

[25] Y. Kabashima, J. Phys. A 36, 11111 (2003).

[26] J. R. L. de Almeida and D. J. Thouless, J. Phys. A 11, 983 (1978).

[27] The definition of $\mu_{i}$ is because of the numerical convenience. Another definition such as $\mu_{i}=\overline{\operatorname{sign}\left(\sum_{j=1}^{N} S_{j} / N\right) S_{i}}$ does not change the results.

[28] The correlation between responses to $\delta \boldsymbol{h}_{i}$ for $i>N_{T}$ is small compared with those of the target spins. As will be discussed later, this is a consequence of the evolution under the fitness defined on the target spins.

[29] D. C. Mattis, Phys. Lett. 56A, 421 (1976).
[30] D. J. Amit, Modeling Brain Function: The World of Attractor Neural Network (Cambridge University Press, Cambridge, 1992).

[31] J. J. Hopfield, Proc. Natl. Acad. Sci. U.S.A. 81, 3088 (1984).

[32] See Supplemental Material at http://link.aps.org/ supplemental/10.1103/PhysRevLett.124.218101 for Fig. 1 showing $\rho$ dependence of the embedded pattern, and Fig. 2 for $\epsilon$ dependence of $d$ over different values of target ratio $\rho$ at $T=1$. Although a strong correlation at $\epsilon=0$ is observed for any $\rho$, the relationship is not robust to noise $\epsilon$ as $\rho$ increases. The existence of redundant spins other than targets is relevant to robustness and dimension reduction.

[33] Approximations Eqs. (9) and (10) are relatively inaccurate for components with small local magnetization, in the sense that they are sensitive to the correction of first eigenmodes by taking higher modes into account. Therefore, the correlations between $\left\{\chi_{i j}\right\}$ and $\left\{\chi_{i k}\right\}$ are observed for components whose local magnetizations are sufficiently large, including target components.

[34] S. Ciliberti, O. C. Martin, and A. Wagner, PLoS Comput. Biol. 3, e15 (2007).

[35] K. Kaneko, PLoS One 2, e434 (2007).

[36] S. Saito, M. Sasai, and T. Yomo, Proc. Natl. Acad. Sci. U.S.A. 94, 11324 (1997).

[37] Y. Togashi and A.S. Mikhailov, Proc. Natl. Acad. Sci. U.S.A. 104, 8697 (2007).

[38] O. Rivoire, Phys. Rev. E 100, 032411 (2019).

[39] K. Husain and A. Murugan, arXiv:1910.09491.

[40] R. Mizuuchi, K. Usui, and N. Ichihashi, RNA 26, 83 (2020).

[41] S. A. Kauffman, The Origins of Order: Self-Organization and Selection in Evolution (Oxford University Press, New York, 1993).

[42] B. Derrida and Y. Pomeau, Europhys. Lett. 1, 45 (1986).

[43] E. Mjolsness, D. H. Sharp, and J. Reinitz, J. Theor. Biol. 152, 429 (1991). 